\title{
The management of fragility fractures of the hip: a quality assessment project
}

\author{
N Kauta, ${ }^{1}$ M Held, ${ }^{2}$ S Dlamini, ${ }^{3}$ S Kalula,${ }^{4}$ I Ross, ${ }^{5}$ G Kalla,,${ }^{6}$ S Maqungo ${ }^{7}$ \\ MBChB, MMed(UCT), FC Orth(SA), Department of Orthopaedics \\ FC Orth(SA), MMed(UCT), PhD, Department of Orthopaedic Surgery \\ MBChB, FCP(SA), Department of Infectious Diseases \\ MBChB, FRCP, MMed(UCT) PhD, Department of Geriatrics \\ MBChB, FCP(SA), FRCP, PhD, Department of Endocrinology \\ MBChB, FCP(SA) MD, Department of Rheumatology \\ MBChB, FC Orth(SA), MMed, PGDipHPE, Department of Orthopaedics \\ All from Groote Schuur Hospital, University of Cape Town, Cape Town, South Africa
}

Corresponding author: Dr N Kauta, PO Box 1293, Mowbray, 7700, Email: Jimmykauta@gmail.com, Cell: 0718962589, Work: 021404 5108

\begin{abstract}
Fragility fractures of the hip (FFH) constitute the most serious complication of osteoporosis, carrying a mortality rate of up to $30 \%$ in the first year after injury. Less than $50 \%$ of affected patients will regain their pre-injury activity level.

Surgical fixation of the hip fracture within 48 hours of injury, multimodal pain management, deep vein thrombo-prophylaxis, early physical therapy and simultaneous management of osteoporosis and frailty in a multidisciplinary approach constitute the standard of care for FFH to keep the mortality and morbidity rates as low as possible and prevent future fragility fractures.
\end{abstract}

Aim: To assess the standard of care of FFH at our institution and determine areas of management which require more attention and improvement.

Methods: A retrospective review of clinical and radiographic records of all patients admitted at our institution for FFH from $1 \mathrm{January}$ 2014 to 31 December 2014.

The waiting time from admission to surgical fixation of the hip fracture, pain control and thrombo-prophylaxis strategies, rate of geriatric referrals and extent of osteoporosis management were assessed.

Results: One-hundred-and-thirteen FFH were admitted from 1 January to 31 December 2014; 98 clinical records and 98 pelvis radiographs were included in the study. The other 15 clinical records were incomplete and were therefore excluded.

The average waiting time from admission to surgery was 49 hours (range 9-120). All patients received low dose morphine, paracetamol and tramadol for perioperative pain control. Low molecular weight heparin and compression stockings were prescribed for thrombo-prophylaxis in all patients.

Only two (2.04\%) patients had some osteoporosis investigations ordered and none of the patients were referred to the geriatric department, and none were formally treated for osteoporosis.

Conclusion: While the waiting time from admission to surgery was largely within the recommended time frame, there were no signs of a multidisciplinary approach to the management of FFH at our institution, leaving osteoporosis and frailty largely untreated.

Key words: fragility fractures, hip, geriatric hip fractures, standard of care for hip fractures, osteoporosis, frailty care

Citation: Kauta N, Held M, Dlamini S, Kalula S, Ross I, Kalla G, Maqungo S. The management of fragility fractures of the hip: A quality assessment project. SAOJ 2017;16(3):41-45. DOI 10.17159/2309-8309/2017/v16n3a5

Editor: Prof Anton Schepers, University of the Witwatersrand

Received: August 2016 Accepted: December 2016 Published: August 2017

Copyright: @ 2017 Kauta N, et al. This is an open-access article distributed under the terms of the Creative Commons Attribution Licence, which permits unrestricted use, distribution and reproduction in any medium, provided the original author and source are credited.

Funding: The authors declare they received no funding for its research.

Conflict of interest: The authors declare they have no conflict of interest in the writing of this study. 


\section{Introduction}

A hip fracture that is sustained following a minimal trauma such as a fall from standing height is defined as a fragility fracture of the hip (FFH). Fragility fractures of the hip are caused by osteoporosis in its various forms and constitute the most severe complication of osteoporosis because of the associated high mortality and morbidity rates. ${ }^{1}$

The incidence of FFH is expected to continue rising due to the ever-increasing life expectancy seen in developed and emerging countries across the globe. ${ }^{2}$

It is estimated that one in three postmenopausal women and one in five men over the age of 75 years will suffer an FFH in their lifetime. The rising global prevalence of $\mathrm{FFH}$ is estimated to reach 2.6 million in 2025 and 6.3 million by year $2050 .^{2}$

FFH carry a mortality rate of up to $30 \%$ in the first year after injury and are associated with a post-injury decline in patients' level of activity in more than $50 \%$ of cases. ${ }^{2,3}$ These factors make FFH the most serious complication of osteoporosis.

The understanding that patients affected by FFH often present with a background of heavy medical co-morbidities and medical frailty has led to the development of strict management guidelines with emphasis on a multidisciplinary approach to improve survival rate and clinical outcome after an FFH.

The American Academy of Orthopaedic Surgeons clinical practice guidelines stipulate that surgical fixation of the hip fracture within 48 hours of injury coupled with multimodal pain treatment, deep vein thrombosis (DVT) prophylaxis, frailty and osteoporosis management will ensure a positive clinical outcome. The presurgery waiting time should only be allowed for medical management of unstable or uncontrolled co-morbid conditions. ${ }^{4}$

Studies have shown a tendency to neglect the management of osteoporosis and frailty in patients who have been treated for FFH at various orthopaedic centres across the world, with only $2 \%$ to $8 \%$ of these patients being referred for osteoporosis treatment. 5,6

Osteoporosis is broadly classified in two major groups: ${ }^{7}$

- Primary osteoporosis: This group is further classified in two types. Type I osteoporosis defines the group of postmenopausal osteoporosis and type II defines senile osteoporosis which affects patients after the age of 75 years. ${ }^{8,9}$

- Secondary osteoporosis: This group defines osteoporosis due to a medical condition such as inflammatory and connective tissue disorders (rheumatoid arthritis, systemic lupus erythematosus, etc.) or malignancies such as multiple myeloma, endocrinopathies such as hyperparathyroidism from various causes, hypogonadism, celiac disease and chronic use of certain drugs such as steroids. ${ }^{7,8}$

It is vitally important that osteoporosis, the main aetiological factor of $\mathrm{FFH}$, be appropriately investigated and classified to initiate suitable treatment.

Alongside osteoporosis many other risk factors play a significant role in causing FFH. These factors need to be identified at the initial admission and appropriate measures taken to prevent further falls and fractures. These risk factors include cognitive impairment, visual impairment, balance and gait disorders, sarcopaenia, use of neuroactive medication, history of maternal FFH, patient's history of any fragility fracture. 8,10

An integrated and multidisciplinary approach is highly desirable to ensure that medical co-morbidities are stabilised, the acute injury is adequately treated and, simultaneously, osteoporosis is investigated and its treatment initiated before the patient is discharged. This comprehensive approach will decrease mortality and morbidity and ensure secondary prevention of subsequent fragility fractures.

The purpose of this study was to assess the standard of care of FFH at our Institution and identify areas of management that require improvement.

\section{Patients and methods}

A retrospective review of clinical and radiographic records was conducted of all patients admitted with an FFH at our level 1 trauma unit from 1 January 2014 to 31 December 2014.

Patients' details were obtained from the admissions records and matched with details on the surgical database. It was possible to retrieve their folders from the records department and their radiographs were available on the digital PACS (picture archiving and communication system).

Only fractures sustained due to falling from standing height or lower (bed or chair) with radiographic osteopaenia were included. Pathological and high energy fractures were excluded. Incomplete clinical records were excluded.

Clinical records were examined to determine the waiting time from admission to surgery, pain control and thrombo-prophylaxis strategies, the rate of geriatric referrals and the extent of evaluation and management of osteoporosis. Formal evaluation of osteoporosis meant that serum vitamin D, calcium levels, erythrocyte sedimentation rate, thyroid function test and parathyroid hormone level were all requested with or without a DEXA scan.

Ethical approval was obtained from our institution's Human Research Ethics Committee.

\section{Results}

We admitted 113 FFH from 1 January to 31 December 2014; 98 clinical records and 98 pelvis radiographs were included in the study. The other 15 clinical records were incomplete and therefore excluded. Eighty-three patients (84.7\%) were females and $15(15.3 \%)$ were males (Figure 1). The mean age was 71.6 years (range 40-93) (Figure 2). The average waiting time from admission to surgery was 49 hours (range 9-120). All patients had low dose morphine, tramadol and paracetamol prescribed for pain control. They all had low molecular weight heparin 40 mg subcutaneously and compression stockings prescription for thrombo-prophylaxis. Two patients (2.04\%) had a DEXA scan done for bone mineral density assessment but none of the patients were formally investigated for osteoporosis. No patient had a vitamin D or calcium prescription in hospital or on subsequent visits. Bisphosphonates were not prescribed for any of the patients and none of the patients were referred to the geriatric department (Figure 3).

Fifteen (15.3\%) patients presented with a history of a previous fragility fracture of the contra-lateral hip and two of them had two previous major fragility fractures (spine and proximal humerus fractures) without any previous assessment or management of osteoporosis.

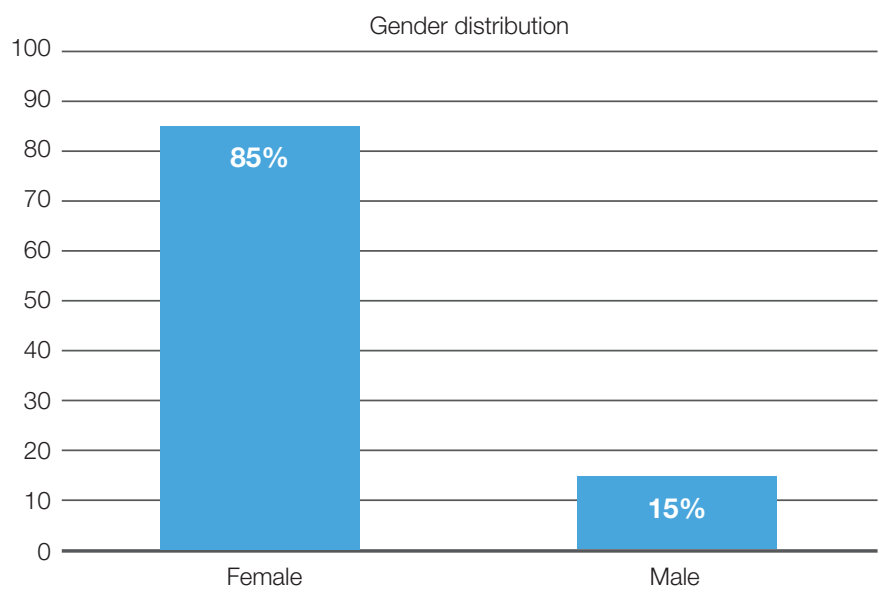

Figure 1. Gender distribution - $85 \%$ of patients were female and $15 \%$ male 


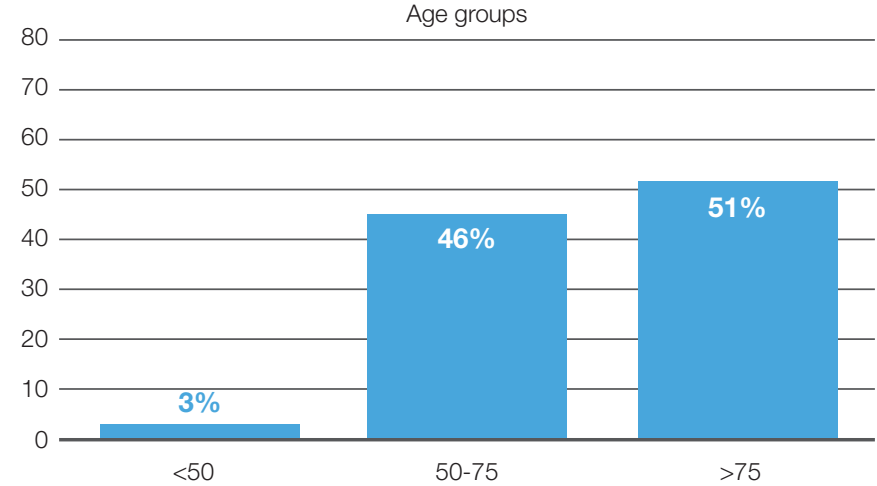

Figure 2. Age distribution - the majority of patients were older than 75 years of age

\section{Discussion}

The results of this study have emphasised that fragility fractures are not only an injury of the female gender but that males also can be affected. In our study $15 \%$ of cases were males. On further analysis $3 \%$ of the patients who were under 50 years of age were males. Despite their lower incidence of fragility fractures male patients tend to have poorer outcomes compared to postmenopausal women. ${ }^{11}$

The aim of treatment of FFH is to restore the patient's pre-injury activity level while minimising morbidity and mortality and laying out a plan for secondary prevention of future fragility fractures. To achieve this aim, a multidisciplinary approach is required to cater for the wide variety of physiological disturbances that are often found in these patients.

The emergency physician would usually refer these patients straight to the orthopaedic surgeon and the later becomes a key player in the multidisciplinary team that will be looking after these patients.

Ekman, in his review article, gives a check list of management priorities that the orthopaedic surgeon must satisfy while treating FFH. This checklist ensures that patients receive a holistic treatment for a positive outcome. ${ }^{12}$

The multidisciplinary approach is aimed at reducing peri-operative complications seen in patients being treated for $\mathrm{FFH}$. These complications include DVT, pulmonary infections, delirium, decubitus pressure ulcers and cardiovascular events.

The current recommendations for the treatment of FFH involve a specialised pain management strategy, thrombo-prophylaxis, early surgery (within 48 hours of admission), referral to geriatricians, physical therapists, dieticians and endocrinologists for a multidisciplinary care. ${ }^{4}$
The results of our study show that there was urgency in the surgical management of $\mathrm{FFH}$ with an average waiting time of 49 hours from admission to surgery. Patients who did not require advanced cardiopulmonary investigations for their pre-operative work up could get to theatre within 9 hours of admission into hospital. However, patients who required extensive cardiopulmonary evaluation and stabilisation could wait up to 120 hours. Our waiting time to surgery signals that our casualty personnel, orthopaedic surgeons and anaesthetists understand the urgency to surgically stabilise these fractures and allow early rehabilitation.

Special investigations such as cardiac echography and lung function test constituted the major reasons for delayed surgical fixation of the hip fracture. Patients admitted on a Friday evening would wait till the next Monday for a cardiac echography or a lung function test to be done if necessitated by their cardiopulmonary conditions.

In a prospective observational study Pioli reported that the oneyear mortality risk increased by $12 \%$ per day of surgical delay. ${ }^{13}$ It is therefore imperative that all unnecessary delays to surgery be proactively avoided to prevent morbidity and mortality.

All our patients had low-dose morphine, tramadol and paracetamol prescribed for pain control. This conservative approach undertreats pain and predisposes to pain-induced delirium. In a single centre study, initiation of a standardised pain control programme including the pre-operative use of femoral nerve blocks, avoidance of general anaesthesia and liberal use of oral intakes (food and oral fluids) pre-operatively resulted in much lower peri-operative complications (pneumonia, urinary tract infection, delirium) compared to preprogramme rates. ${ }^{14}$

DVT is another major complication that must be prevented in this fragile population of patients. A meta-analysis of prospective cohorts in Asian patients with hip fractures revealed that $26 \%$ of patients not on DVT prophylaxis developed a post-operative DVT. ${ }^{15}$ It is therefore recommended that all patients receive effective DVT prophylaxis. All our patients had low molecular weight heparin (enoxaparin $40 \mathrm{mg}$ once daily subcutaneously) prescribed for DVT prophylaxis. This was combined with a prescription of compression stockings for all patients.

Fifty-one per cent of reviewed cases were patients above the age of 75 years. In this age group, the incidence of fragility fracture rises significantly. ${ }^{16}$ This is probably due to a combination of frailty and senile osteoporosis. These patients need to be seen by a geriatrician who will optimise their medical condition while the orthopaedic surgeon focuses on surgical treatment. None of our patients were referred to a geriatrician.

A quarter of patients admitted with fragility fractures will develop delirium. ${ }^{17}$ Predisposing factors for delirium include male gender, multiple co-morbidities, low body mass index (BMI), prolonged

Measures of standard of care

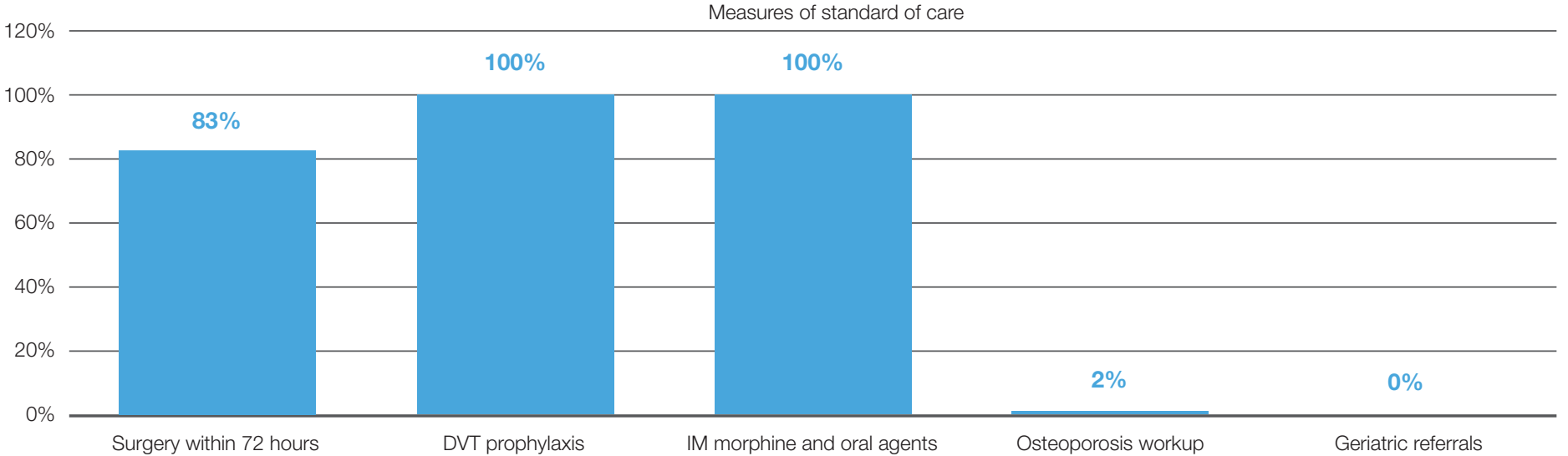

Figure 3. Measures of standard of care: only $2 \%$ of patients investigated for osteoporosis and no geriatric referrals 
surgery and general anaesthesia. ${ }^{17}$ Post-operative delirium is usually transient but it can persist for more than four weeks in $20 \%$ of patients. Delirium is associated with lower functional recovery and higher mortality rate at one year. ${ }^{18}$ Therefore the prevention of delirium becomes of paramount importance.

The literature supports that early referral to geriatricians is associated with a lower rate of post-operative delirium. ${ }^{19,20}$

Where the service of a geriatrician is lacking, a specialist physician with an interest in the field of geriatrics can be consulted for the management of these patients.

By definition, an FFH implies a diagnosis of osteoporosis. According to the $\mathrm{WHO}$ criteria, a diagnosis of osteoporosis is made when a patient has a T-score lower than -2.5 or when a patient presents with a typical fragility fracture defined as a fracture occurring after a fall from standing height or lower and has osteopaenia on plain radiographs. ${ }^{8}$ This definition excludes pathological fractures due to malignancies and infections. Osteoporosis must be investigated, classified and appropriately treated for every patient presenting with an $\mathrm{FFH}$.

In our study only two out of 98 patients had a DEXA scan done with no other osteoporosis investigations ordered. None of our patients were treated for osteoporosis. This finding outlines the fact that orthopaedic surgeons satisfy themselves with a well-performed fracture fixation as seen on post-operative radiographs and forget to facilitate or initiate the management of frailty and osteoporosis.

Similar studies conducted in Europe and in the United States have reported the same trend. Very few patients presenting with FFH will be treated for osteoporosis. Rabenda et al. reported that only $4.6 \%$ of patients with FFH were fully treated for osteoporosis in their study ${ }^{5}$ and Jennings reported a $2 \%$ rate of osteoporosis treatment in patients admitted for $\mathrm{FFH} .{ }^{6}$

Osteoporosis work-up starts with a DEXA scan which provides the patient's T-score for the diagnosis and a baseline bone mineral density (BMD) to monitor response to treatment. However, the lack of a DEXA scan should not hinder osteoporosis treatment for patients presenting with an FFH.

Laboratory investigations will help exclude any possible cause of secondary osteoporosis and the following tests should be requested for all patients under investigation for osteoporosis: ${ }^{7,21}$

- Erythrocyte sedimentation rate (ESR)

- Thyroid function test

- Serum parathyroid hormone (PTH) level

- Serum calcium level

- Vitamin D level

- Protein electrophoresis for a myeloma screen when clinically suspected.

The management plan will differ between the two main forms of osteoporosis, namely: primary and secondary osteoporosis.

General nutritional and lifestyle modifications will apply to both primary and secondary osteoporosis and include regular physical activity, and adequate intake of calcium, vitamin D and proteins.

A daily intake of at least $1.2 \mathrm{~g}$ of calcium and $800 \mathrm{IU}$ of vitamin D has been shown to reduce the relative risk of falls and fragility fractures more in the institutionalised elderly patients than in the community dwellers. ${ }^{1,22}$

Sarcopaenia is being increasingly recognised as an independent risk factor for falls and fragility fractures. ${ }^{23}$ Physiotherapists and dieticians work together to correct sarcopaenia and optimise physical performance of these patients for a better clinical outcome.

Smoking needs to be stopped and alcohol consumption moderated.

Pharmacological treatment will depend on the type of primary osteoporosis being addressed.

Postmenopausal osteoporosis may benefit from hormone replacement therapy early on but as senile osteoporosis sets in bisphosphonates therapy will be required to maintain adequate bone mineral density.
Once all causes of secondary osteoporosis have been excluded, bisphosphonate therapy is indicated. High-risk patients as determined by the applicable Fracture Risk Assessment Tool (FRAX index) are also eligible for treatment. ${ }^{22,24}$ The FRAX tool combines patient's specific BMD and clinical risk factors such as age, gender, $\mathrm{BMI}$, history of fragility fractures, history of maternal hip fracture and current smoking status to calculate a ten-year risk of hip or other osteoporosis-related fractures for the patient being assessed. This tool is country specific. ${ }^{24}$ Most commonly, oral alendronate $70 \mathrm{mg}$ weekly or zolendronic acid $5 \mathrm{mg}$ IV yearly is prescribed. ${ }^{25}$

Alendronate has been proven to reduce the vertebral fracture risk by $60 \%$ and hip fracture risk by $30 \% .{ }^{25}$ Zolendronic acid once yearly showed a $70 \%$ risk reduction of morphometric vertebral fractures and $41 \%$ risk reduction of hip fractures at 36 months. ${ }^{26}$

The most debated side effects of bisphosphonates are atypical femur fractures, osteonecrosis of the jaw and delayed or impaired fracture healing. ${ }^{22,25}$

The rate of atypical femur fractures varies in the literature but the known fact is that the incidence shows an exponential increase with the increase in the number of years of continuous bisphosphonates treatment. ${ }^{25}$

Osteoclastic activity remains inhibited for a few years after discontinuation of bisphosphonates use. This allows for a 'drug holiday' to minimise the rate of atypical fractures. ${ }^{26}$

In the setting of osteoporosis treatment, the rate of osteonecrosis of the jaw is much lower than that of atypical femoral fractures. ${ }^{25,26}$

A meta-analysis of eight randomised controlled trials found that bisphosphonate treatment did not impair or delay fracture healing in the short term (three months) or long term (more than 12 months). ${ }^{27}$

Other recent and not widely available pharmacological agents for the treatment of osteoporosis include parathyroid hormone (Forteo, Teriparatide), denosumab (Prolia), strontium ranelate (Protos) and cathepsin $\mathrm{K}$ inhibitors. ${ }^{28-31}$

To simply provide a sound and early surgical fixation of the hip fracture and not address the underlying osteoporosis, frailty and certain possible causes of falls is in a way failing to provide the holistic and comprehensive management that these patients need.

The orthopaedic surgeon is most often the first specialist to see these patients and it is his or her responsibility to make early and appropriate referrals to geriatricians, physicians, endocrinologists, physical therapists and dieticians. These other team players will help optimise patients' medical condition to allow early surgery, early rehabilitation, optimal investigations, prevention and treatment of peri-operative complications and lay out a plan for osteoporosis treatment.

The literature shows that less than $10 \%$ of patients presenting to major trauma centres with FFH are fully evaluated and managed for osteoporosis. This outlines the need to increase awareness of osteoporosis management in orthopaedic trauma centres worldwide. ${ }^{4,6,32}$

This study was the first step of our quality assessment project for the management of FFH at our institution. It has led to the development of a strict management protocol and the genesis of an ortho-geriatric unit called GOGO (Geriatric Orthopaedic and Generalised Osteoporosis) that functions as our fracture liaison service for a focused and improved care for FFH as the primary target.

The ortho-geriatric unit aims at providing an integrated service where orthopaedic surgeons and geriatricians provide integrated care without the frustration of lengthy telephonic referrals. Geriatricians see these patients as their own patients and not as orthopaedic patients.

There are various models of integrated fragility fracture care available. Each institution treating these fractures should adopt a suitable model that promotes an integrated multidisciplinary approach and optimal treatment of these fragile patients for secondary prevention of fragility fractures. 


\section{Conclusion}

The vast majority of our patients had their hip fractures surgically fixed within the recommended time frame. Our management approach lacked the multidisciplinary integration that is recommended for these patients. Osteoporosis and frailty were largely not addressed. The results of this study served as a platform to increase awareness of osteoporosis and frailty management as part of a multidisciplinary care for $\mathrm{FFH}$. We have subsequently formed a multidisciplinary unit in an effort to address our treatment deficiencies and to improve these patients' outcomes.

\section{Compliance with ethics guidelines}

Ethical approval was obtained from our institution's Human Research Ethics Committee.

\section{References}

1. Tp IP, Leung J, Kung AWC. Management of osteoporosis in patients hospitalized for hip fractures, Osteoporos Int 2010;21(4): S605-614.

2. Cassim B, Lipschitz S, Paruk F, Tipping B. Recommendations for the acute and long-term medical management of low trauma hip fractures. JEMDSA 2013;18(1):21-32.

3. Cooper C, Campion G, Melton LJ 3rd. Hip fractures in the elderly: a worldwide projection. Osteoporosis Int. 1992;2(6):285-89.

4. Robert KC, Brox WT, Jevsevar DS, Sevarino K. Management of hip fractures in the elderly. J Am Acad Orthop Surg 2015;23:131-37.

5. Rabenda V, Vanoverloop J, Fabri V, Mertens R. Low incidence of anti-osteoporosis treatment after a hip fracture. J Bone Joint Surg Am. 2008;90:2142-48.

6. Jennings LA, Andrew D, Maselli J, et al. Missed opportunities for osteoporosis treatment in patients hospitalized for hip fractures. J Am Geriatr Soc. 2010;58(4):650-57.

7. NIH Consensus Panel Development on Osteoporosis Prevention, Diagnosis and therapy. JAMA 2001; 285(6):785-95.

8. Lorentzon M, Cummings SR. Osteoporosis: the evolution of a diagnosis. J Intern Med 2015;277:650-61.

9. Riggs BL, Melton LJ 3rd. Evidence for two distinct syndromes of involutional osteoporosis. Am J Med 1983;75:899-901.

10. Tinetti, ME, Speechley M, Ginter SF. Risk factors for falls among elderly persons living in the community. N Engl J Med 1988; 319:1701-07.

11. Peggy MC. Gender differences in osteoporosis and fractures. Clin Orthop Relat Res. 2011;469:1900-1905.

12. Ekman EF. the role of the orthopaedic surgeon in minimizing mortality and morbidity associated with fragility fractures. J Am Acad Orthop Surg 2010;18:278-85.

13. Pioli G. Time to surgery and rehabilitation resources affects outcomes in ortho-geriatric units. Archives of Gerontology and Geriatrics 2012;55:316-22.

14. Borgbjerg FM, Schousboe B, Pedersen SJ, et al. A comprehensive hip fracture program reduces complications rate and mortality, J Am Geriatr Soc 2008;56:1831-38.

15. Kanchanabat B, Stapanavatr W, Meknavin S, Soorapanth C, Sumanasrethakul C, Kanchanasuttirakn P. Systematic review and meta-analysis on the rate of postoperative venous thrombo-embolism in orthopaedic surgery in Asian patients without thrombo-prophylaxis, Br J Surg 2011;98:1356-64.

16. Felsenberg D, Silman AJ, Lunt M, et al. Incidence of vertebral fracture in Europe: results from European Prospective Osteoporosis Study (EPOS). J Bone Miner Res 2002;17:716-24.

17. Lee HB, Mears SC, Rosenberg PB, Leoutsakos J-MS, Gottschalk A, Sieber FE. Predisposing factors for postoperative delirium after hip fracture repair in individuals with and without dementia. J Am Geriatr Soc 2011:59:2306-13.

18. Lee K-H, Ha Y-C, Lee Y-K, Kang H, Koo K-H. Frequency, risk factors, and prognosis of prolonged delirium in elderly after hip fracture surgery. Clin Orthop Relat Res 2011;469:2612-20.

19. Marcantonio ER, Flacker JM, Wright RJ, Resnick NM. Reducing delirium after hip fracture: a randomized trial. J Am Geriatr Soc 2001;49:516-22.
20. Deschodt M, Braes T, Flamaing J, Detroyer E, Broos P, Haentjens P, et al. Preventing delirium in older adults with recent hip fracture through multidisciplinary geriatric consultation. J Am Geriatr Soc 2012;60:733-39.

21. Strotman P, Lack W, Bernstein M. Evaluation of common fractures of the hip in the elderly. Cuur Geri Rep 2016;5:38-43.

22. Balach T, Paul C, Baldwin, Intravia J. Atypical femur fractures associated with diphosphonate use. J Am Acad Orthop Surg 2015;23:550-57.

23. Bokshan SL, DePasse JM, Daniels AH. Sarcopenia in orthopaedic surgery. Orthopedics 2016;39(2):e295-e300.

24. Unnanuntana A, Gladnick BP, Lane JM. The assessment of fracture risk. J Bone Joint Surg Am. 2010;92(3):743-53.

25. Reyes C, Hitz M, Alhambra DP. Risks and benefits of bisphosphonates therapies, J. Cell. Biochem 2016;117:20-28.

26. Keneth W, Cathleen S, Jay S, Jonatha D, Carl F, Carlos M. Zolendronic acid and clinical fractures and mortality after hip fracture. N Engl J Med 2007;357:1799-809.

27. Deting X, Fangcai L, Gang C, ShiguiY, Zhijun P. Do bisphosphonates affect bone healing? A meta-analysis of randomized controlled trials. Journal of Orthopaedic Surgery and Research 2014;9:1-45.

28. Morley P, Whitefield JF, Willick GE. Parathyroid hormone: An anabolic treatment for osteoporosis. Curr Pharm Dec 2001;7(8)671-87.

29. Cummings SR, San Martin J, McClung MR, Siris ES, Eastell R, Reid $I R$, et al. Denosumab for prevention of fractures in postmenopausal women with osteoporosis. N Engl J Med 2009;361(8):756-65.

30. Atteritano M, Catalano A, Santoro D, Lasco A, Benvenga S. Effects of strontium ranelate on markers of cardiovascular risk in postmenopausal osteoporotic women. Endocrine 2016;53(1) 305-312.

31. Boonen S, Rosenberg E, Claessens F, Vander Schueren D, Papapoulos S. Inhibition of cathepsin K for treatment of osteoporosis. Curr Osteoporos Rep. 2012 Mar;10(1):73-79.

32. Kaufman JD, Bolander ME, Bunta AD, et al. Barriers and solutions to osteoporosis care in patients with a hip fracture. J Bone Joint Surg Am 2003;85:1837-42. 